\title{
AUTHOR INDEX VOLUME 1 (2000)
}

Bermond, J.-C., Bond, J., Martin, C., Pekeč, A. and Roberts, F.S., Optimal orientations of annular networks Bermond, J.-C., Marshall, S. and Yu, M.-L., Improved bounds for gossiping in mesh bus networks

Bhargava, V.K., see Hossain, E.

Bhattacharjee, P.S., Saha, D. and Mukherjee, A., Intelligent paging strategies for third generation personal communication services networks

Bond, J., see Bermond, J.-C.

Boukerche, A., Load balancing for parallel PCS network simulations

Chen, C. and Hwang, F.K., Equivalent L-shapes of double-loop networks for the degenerate case

Das, S.K., Sen, S.K. and Jayaram, R., D-LBSB: A distributed load balancing algorithm for channel assignment in cellular mobile networks

Datta, A.K., Derby, J.L., Lawrence, J.E. and Tixeuil, S., Stabilizing hierarchical routing

Derby, J.L., see Datta, A.K.

Epema, D.H.J., see Lele, A.M.

Farley, A.M., Fragopoulou, P., Krumme, D., Proskurowski, A. and Richards, D., Multi-source spanning tree problems

Ferreira, A., Goldman, A. and Song, S.W., Broadcasting in bus interconnection networks

Fragopoulou, P., see Farley, A.M.

Goldman, A., see Ferreira, A.

Gonzalez, T.F., Distributed algorithm for multimessage multicasting

Hong, S.J., see Lee, W.Y.

Hossain, E. and Bhargava, V.K., Link-state aware dynamic traffic scheduling for providing predictive QoS in wireless mobile multimedia networks

Hsu, L.-H., see Li, T.-K.

Hsu, W.J., see Loh, P.K.K.

Hwang, F.K., see Chen, C.
1, 1 (2000) 21-46

1, 1 (2000) 1-19

1, 3 (2000) 221-245

1, 3 (2000) 153-171

1, 1 (2000) 21-46

1, 3 (2000) 173-193

$1,1(2000) 47-60$

1, 3 (2000) 195-220

1, 4 (2000) 283-302

1, 4 (2000) 283-302

1, 3 (2000) 247-266

1, 1 (2000) 61-71

1, 2 (2000) 73-94

1, 1 (2000) 61-71

1, 2 (2000) 73-94

1, 4 (2000) 303-314

1, 2 (2000) 95-114

1, 3 (2000) 221-245

1, 2 (2000) 115-134

1, 4 (2000) 315-329

1, 1 (2000) 47-60 
Jayaram, R., see Das, S.K.

Kim, J., see Lee, W.Y.

Krumme, D., see Farley, A.M.

Lawrence, J.E., see Datta, A.K.

Lee, W.Y., Hong, S.J. and Kim, J., On the configuration of switch-based networks with wormhole routing

Lele, A.M., Nandy, S.K. and Epema, D.H.J., Harmony — An architecture for providing quality of service in mobile computing environments

Li, T.-K., Tan, J.J.M., Hsu, L.-H. and Sung, T.-Y., Optimum congested routing strategy on twisted cubes

Liu, W., see Wang, Z.

Loh, P.K.K. and Hsu, W.J., Fault-tolerant communications on hypercube-clusters

Marsan, M.A., see Meo, M.

Marshall, S., see Bermond, J.-C.

Martin, C., see Bermond, J.-C.

Meo, M. and Marsan, M.A., Analysis of hierarchical cellular networks with multimedia services and different user mobility patterns

Mukherjee, A., see Bhattacharjee, P.S.

Nandy, S.K., see Lele, A.M.

Pandurangan, G. and Upfal, E., Static and dynamic evaluation of QoS properties

Pekeč, A., see Bermond, J.-C.

Proskurowski, A., see Farley, A.M.

Richards, D., see Farley, A.M.

Roberts, F.S., see Bermond, J.-C.

Saha, D., see Bhattacharjee, P.S.

Sen, S.K., see Das, S.K.

Shi, B., see Wang, Z.

Song, S.W., see Ferreira, A.

Sung, T.-Y., see Li, T.-K.

Tan, J.J.M., see Li, T.-K.

Tixeuil, S., see Datta, A.K.

Upfal, E., see Pandurangan, G.

Wang, Z., Shi, B. and Liu, W., A distributed dynamic heuristic for delay-constrained least-cost multicast routing $\mathrm{Yu}$, M.-L., see Bermond, J.-C. $\mathbf{1}, 3$ (2000) 195-220

$\mathbf{1}, 2$ (2000) 95-114

$\mathbf{1}, 1$ (2000) 61-71

1, 4 (2000) 283-302

1, 2 (2000) 95-114

1, 3 (2000) 247-266

1, 2 (2000) 115-134

1, 4 (2000) 331-344

1, 4 (2000) 315-329

1, 3 (2000) 267-282

1, 1 (2000) 1-19

1, 1 (2000) 21-46

1, 3 (2000) 267-282

1, 3 (2000) 153-171

1, 3 (2000) 247-266

1, 2 (2000) 135-150

1, 1 (2000) 21-46

1, 1 (2000) 61-71

1, 1 (2000) 61-71

1, 1 (2000) 21-46

1, 3 (2000) 153-171

1, 3 (2000) 195-220

1, 4 (2000) 331-344

1, 2 (2000) 73-94

1, 2 (2000) 115-134

1, 2 (2000) 115-134

1, 4 (2000) 283-302

1, 2 (2000) 135-150

1, 4 (2000) 331-344

1, 1 (2000) 1-19 